\title{
SUPPLIMENTARY INFORMATION
}

Generation and applications of structure envelopes for porous metal-organic frameworks

Andrey A. Yakovenko ${ }^{a *}$, Joseph H. Reibenspies ${ }^{a}$, Nattamai Bhuvanesh ${ }^{\mathrm{a}}$ and Hong-Cai Zhou ${ }^{\mathrm{a} *}$ ${ }^{\text {a}}$ Chemistry Department, Texas A\&M University, College Station, TX, 77843, USA

Correspondence email: ayakovenko@mail.chem.tamu.edu; zhou@mail.chem.tamu.edu 


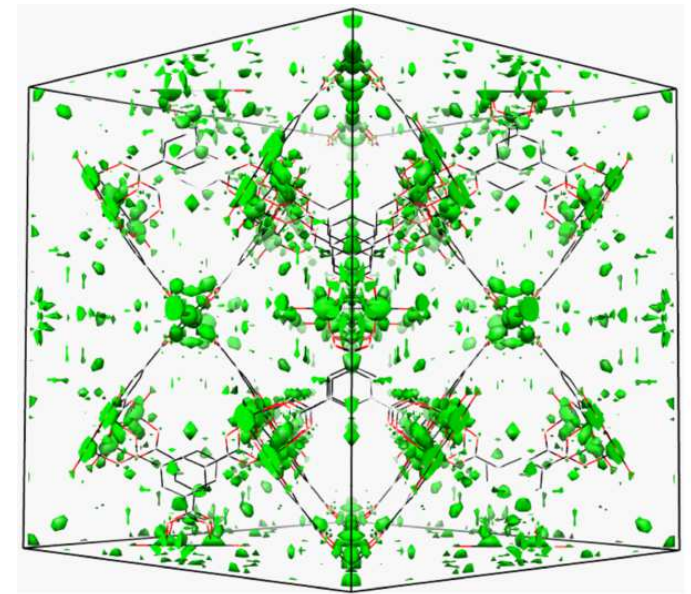

(a)

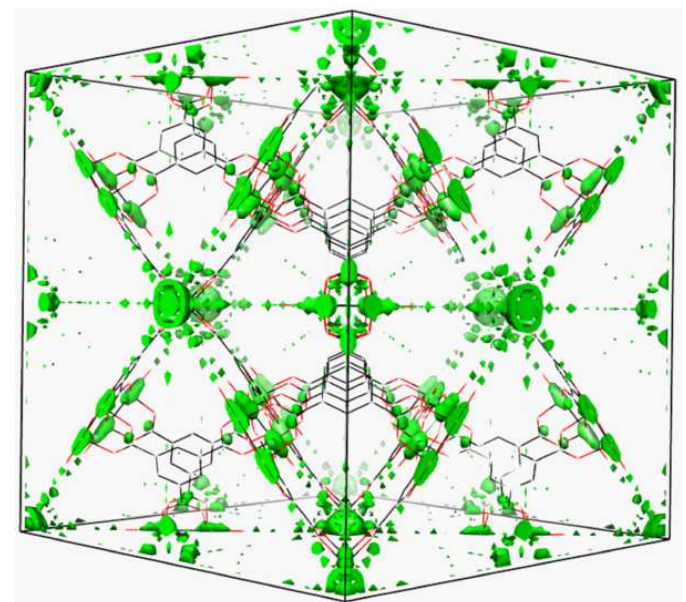

(c)

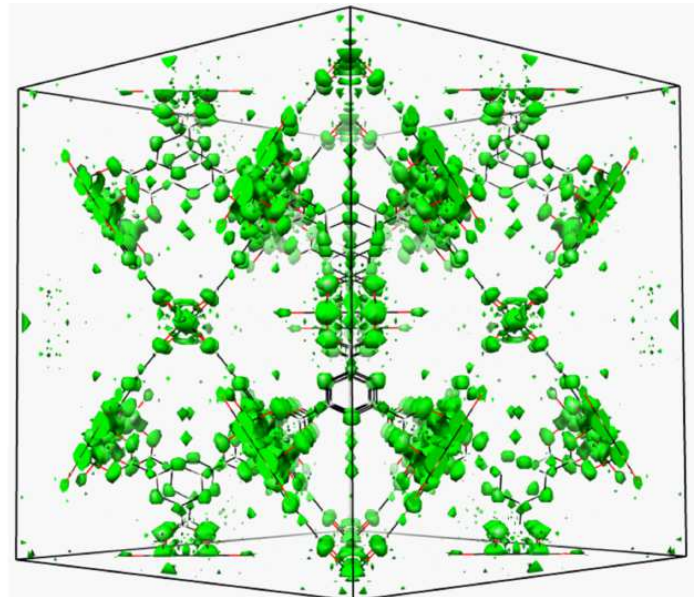

(b)

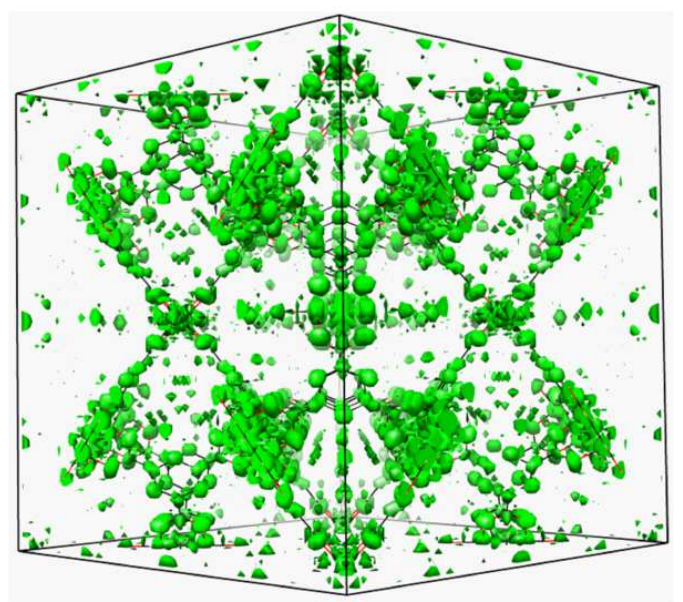

(d)

Figure 1S. Final electron density maps generated for HKUST-1a data set from: (a) CF (20 runs 250 cycles), (b) CF+HM (20 runs 250 cycles), (c) CF (1000 runs 10000 cycles) and (d) CF+HM (50 runs 10000 cycles) calculations. 


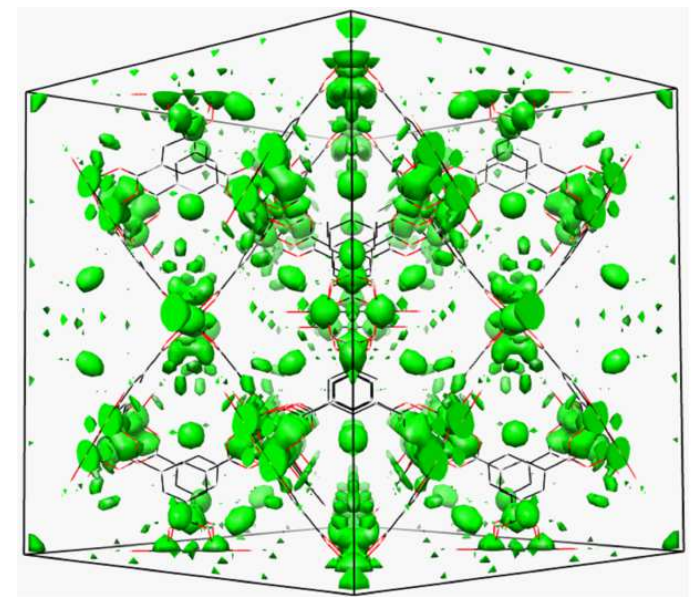

(a)

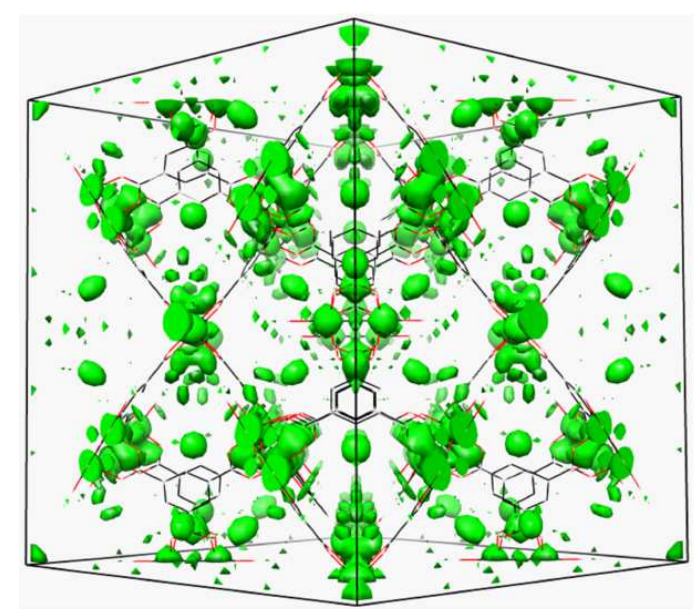

(c)

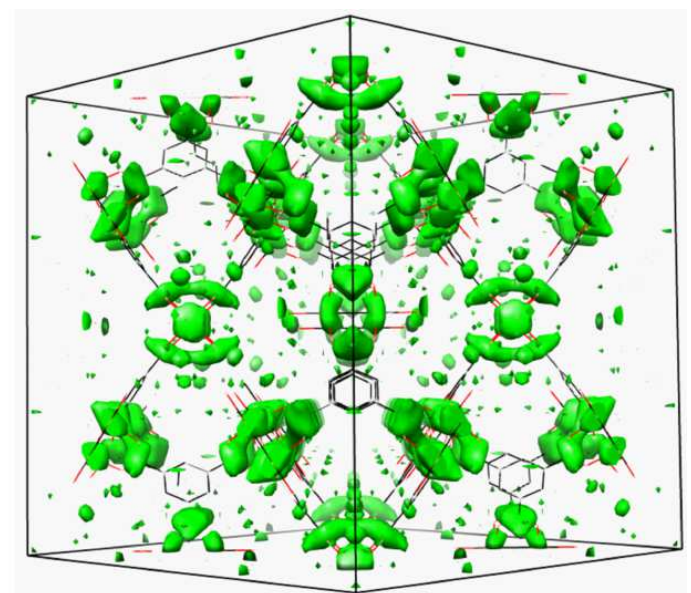

(b)

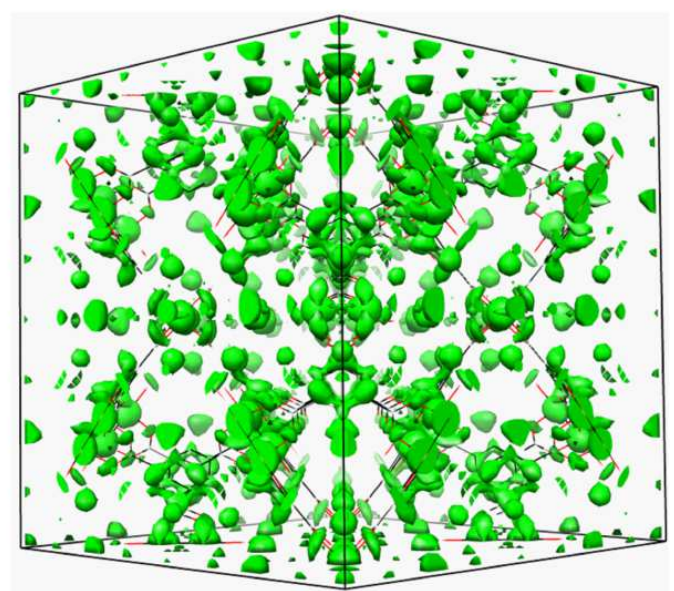

(d)

Figure 2S. Final electron density maps generated for HKUST-1syn data set from: (a) CF (20 runs 500 cycles), (b) CF+HM (20 runs 500 cycles), (c) CF (100 runs 10000 cycles) and (d) CF+HM (100 runs 10000 cycles) calculations. 


\section{Description of Rietveld refinements of HKUST-1a and HKUST-1b}

The structures of HKUST-1a and HKUST-1syn was refined using Jana2006.(Petricek, 2006) Twenty and twelve Legendre polynoms were used to adjust the background, for HKUST-1a and HKUST-1syn respectively, with a Pseudo-Voigt function to determine the peak profile. Two asymmetry parameters, one overall thermal parameter were also applied during refinements. Distance and angle constraints were used during the refinement especially to refine the ligands as a rigid body.

During the refinement we have founds large peaks in the middle of the pores for both HKUST-1 structures. These peaks are probably correspond to the solvent molecules located in the pores. These solvent molecules could not be locaded due to their partial occupancies and lower symmetry. Instead, 2 for HKUST-1a and 15 for HKUST-1syn unique carbon atoms were added at random positions inside the pores and refined subsequently. The final Rietveld refinement plots and the agreement factors are satisfactory and presented on Figure $3 \mathrm{~S}$ and Table 1S.

Table 1S. The Final R-factors for the HKUST-1 samples Rietveld refinements

\begin{tabular}{lccccc}
\hline & $\mathrm{R}_{\mathrm{p}}$ & $\mathrm{R}_{\mathrm{wp}}$ & $\mathrm{R}_{\exp }$ & GOF & $\mathrm{R}_{\text {brag }}$ \\
\hline HKUST-1a & 0.091 & 0.119 & 0.067 & 1.73 & 0.084 \\
HKUST-1syn & 0.077 & 0.097 & 0.059 & 1.64 & 0.068 \\
\hline
\end{tabular}




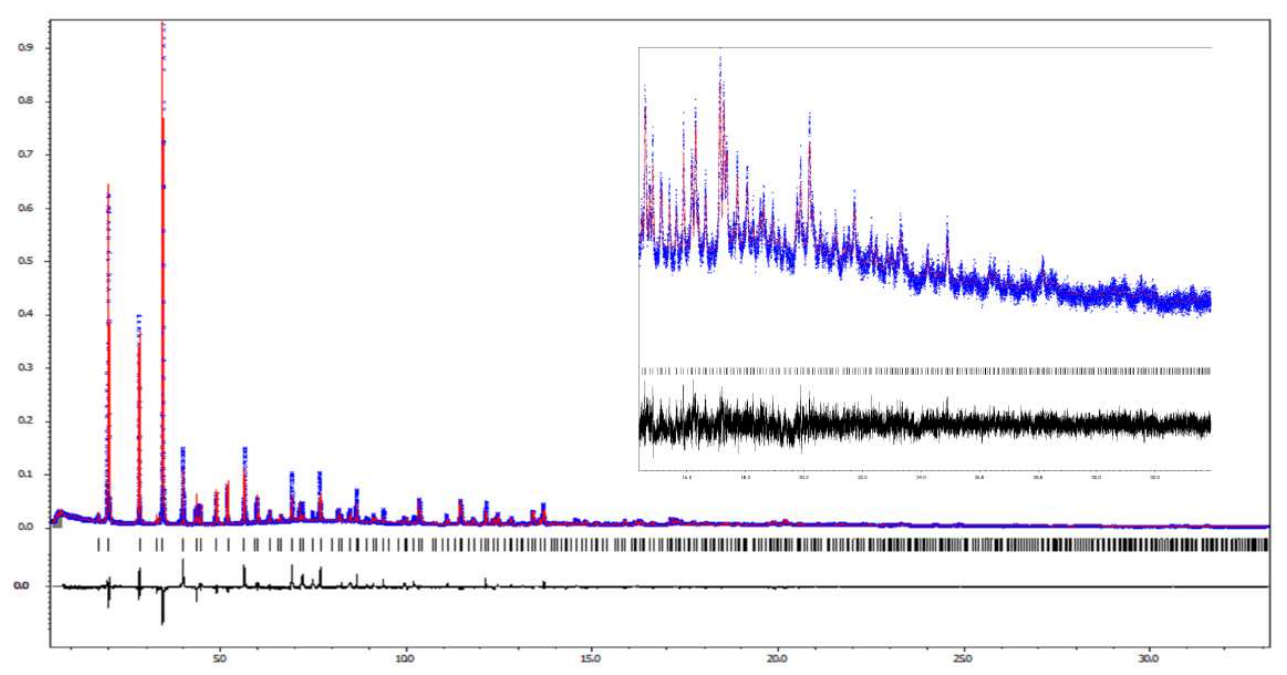

(a)

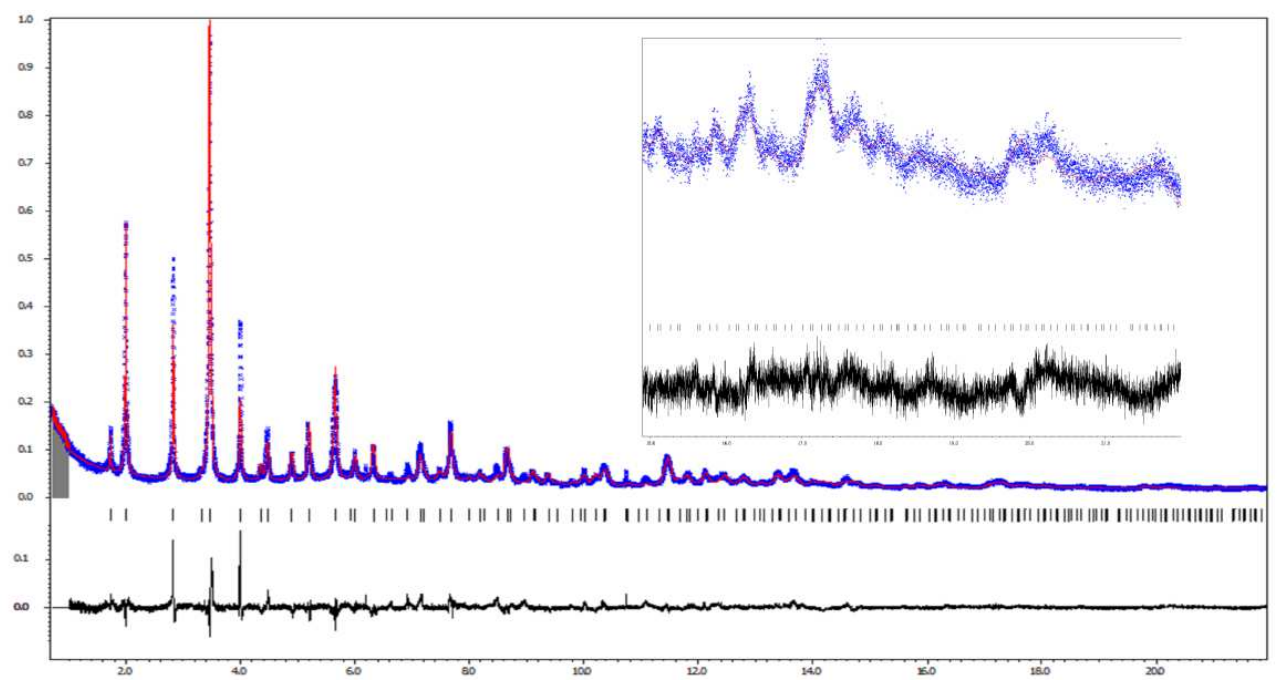

(b)

Figure 3S. Final Rietveld refinement plots for the (a) HKUST-1a and (b) HKUST-1syn samples 


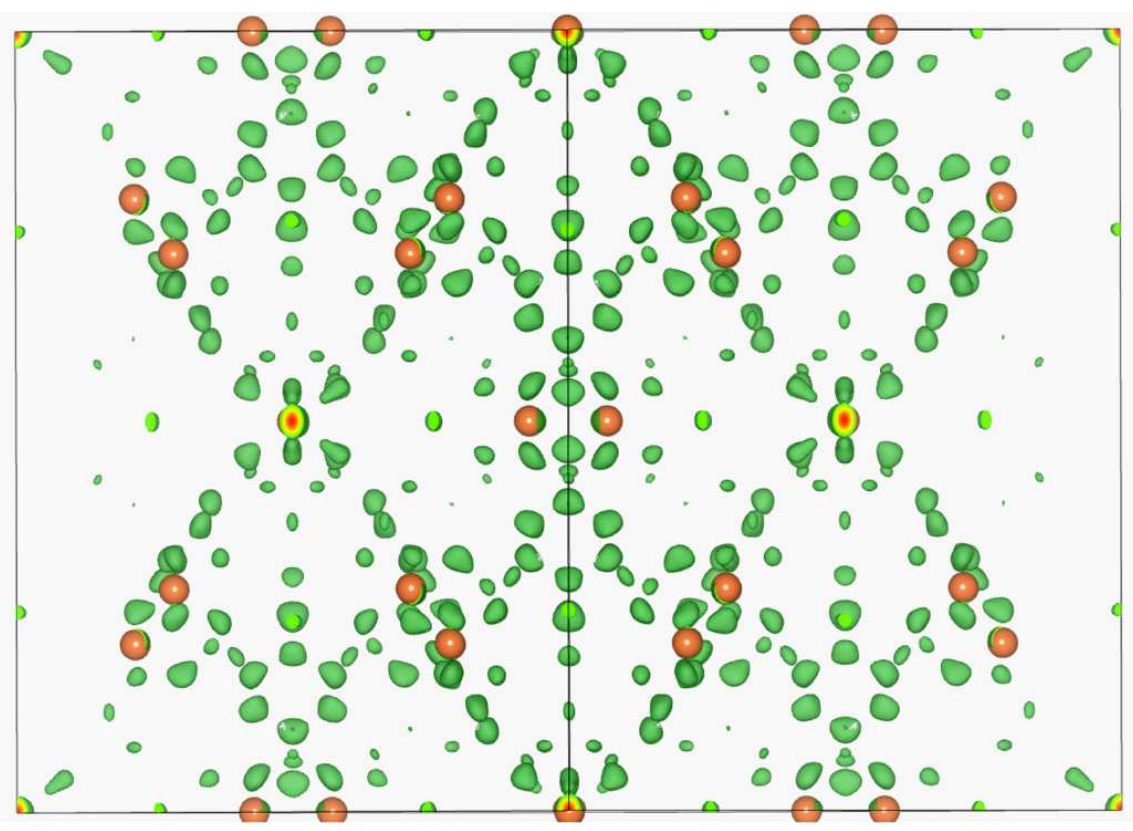

(a)

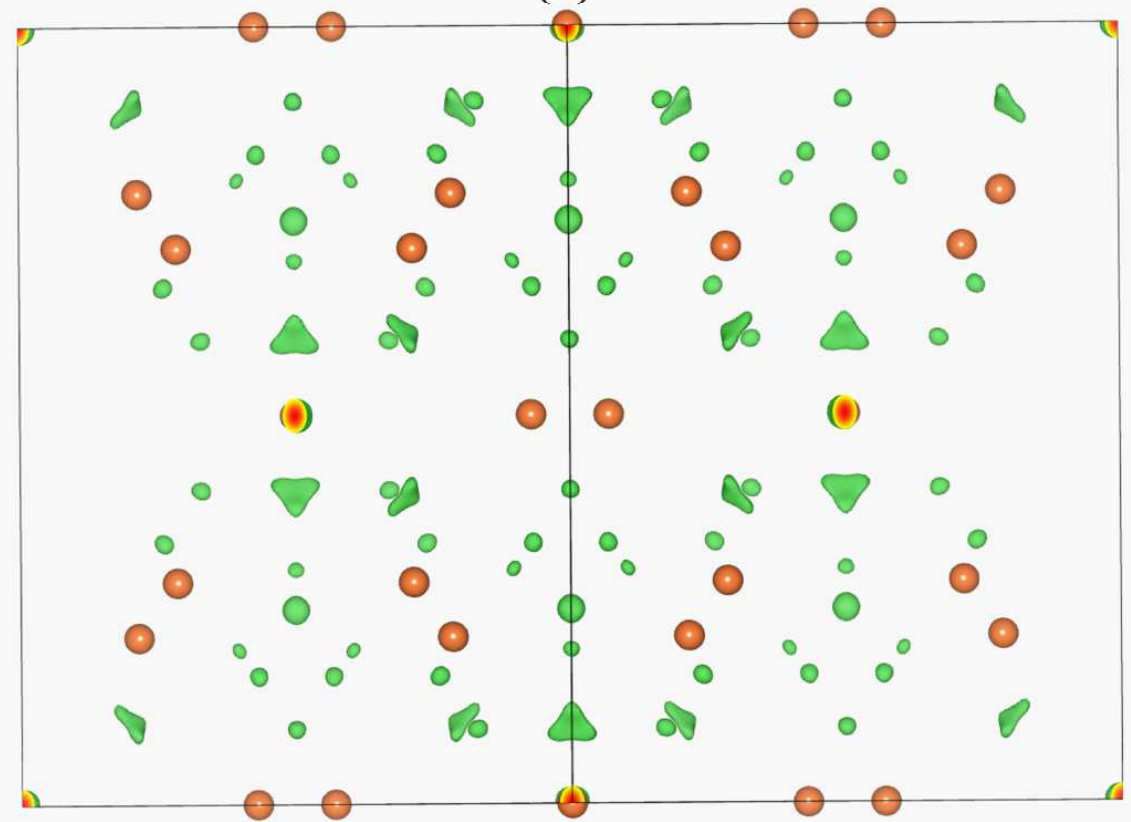

(b)

Figure 4S. Resulting $F_{\text {obs }}-F_{\text {calc }}$ density maps for (a) HKUST-1a and (b) HKUST-1syn samples after introduction only of the $\mathrm{Cu}$ atoms into their Rietveld refinements. 


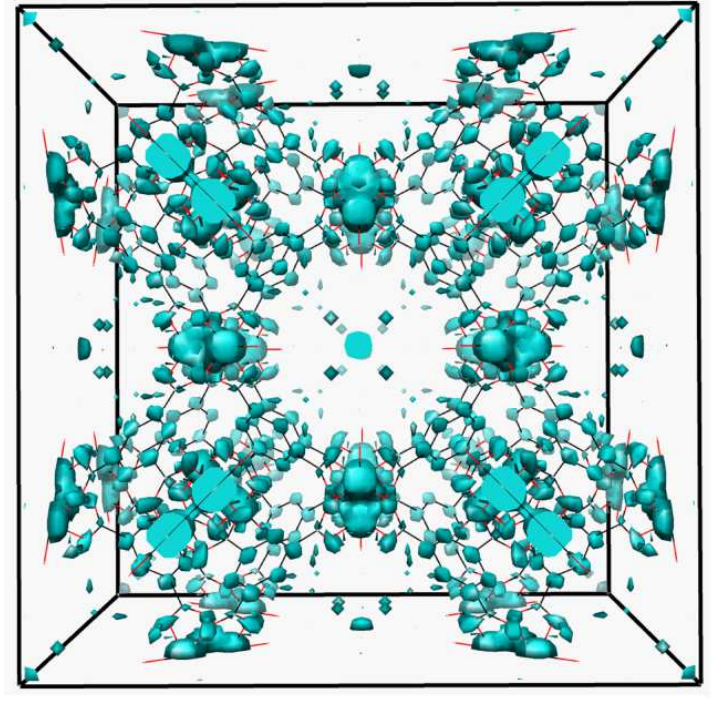

(a)

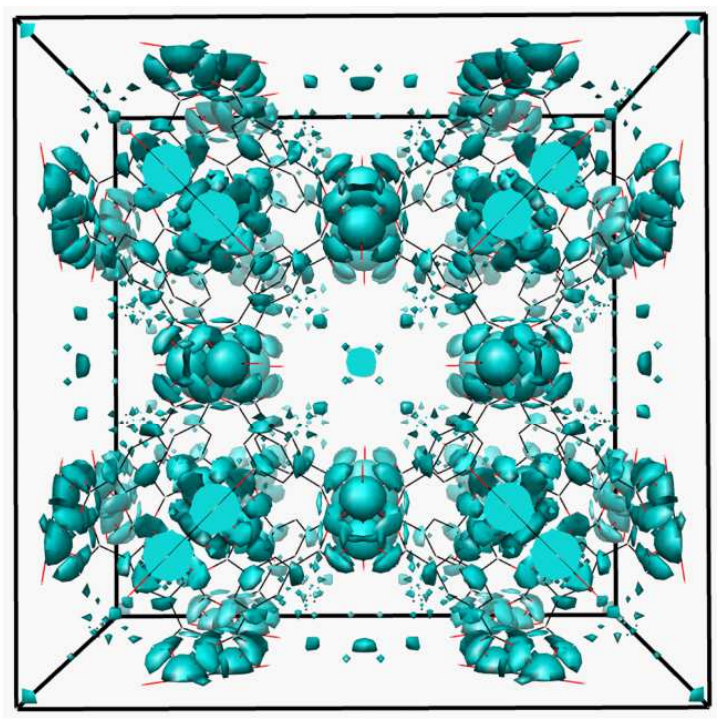

(b)

Figure 5S. Resulting electron density maps for (a) HKUST-1a and (b) HKUST-1syn after CF calculations with Rietveld $\left|F_{o b s}\right|^{2}$.

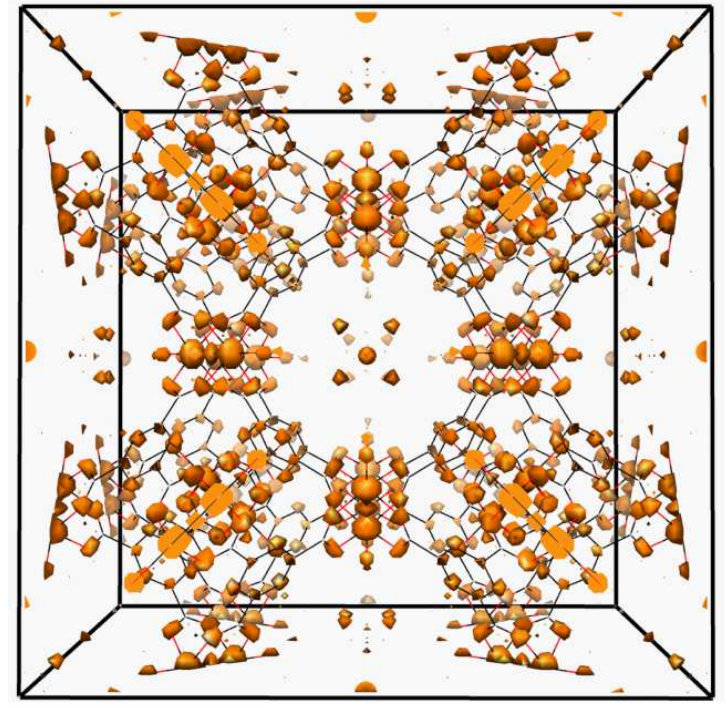

(a)

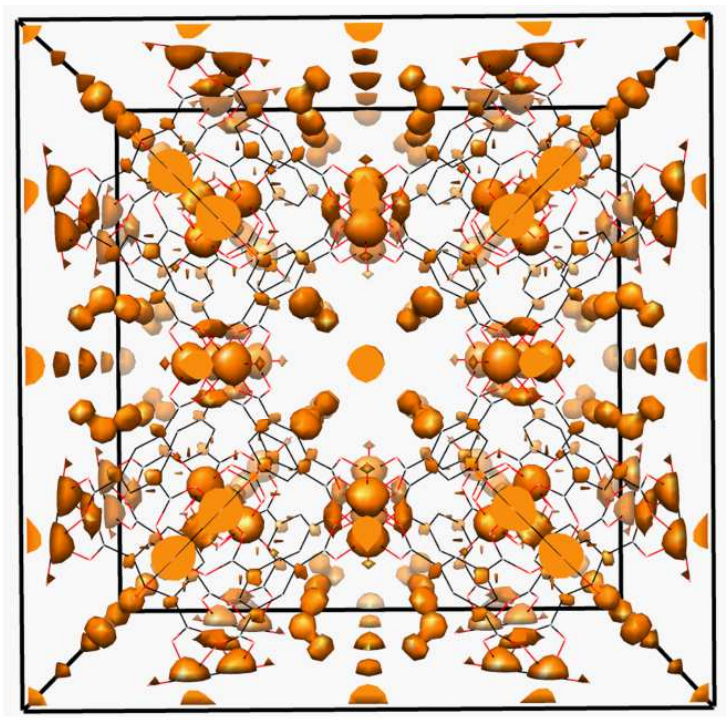

(b)

Figure 6S. Resulting electron density maps for (a) HKUST-1a and (b) HKUST-1syn after introduction of $\mathrm{Cu}$ atom positions directly into $\mathrm{CF}$ calculations 


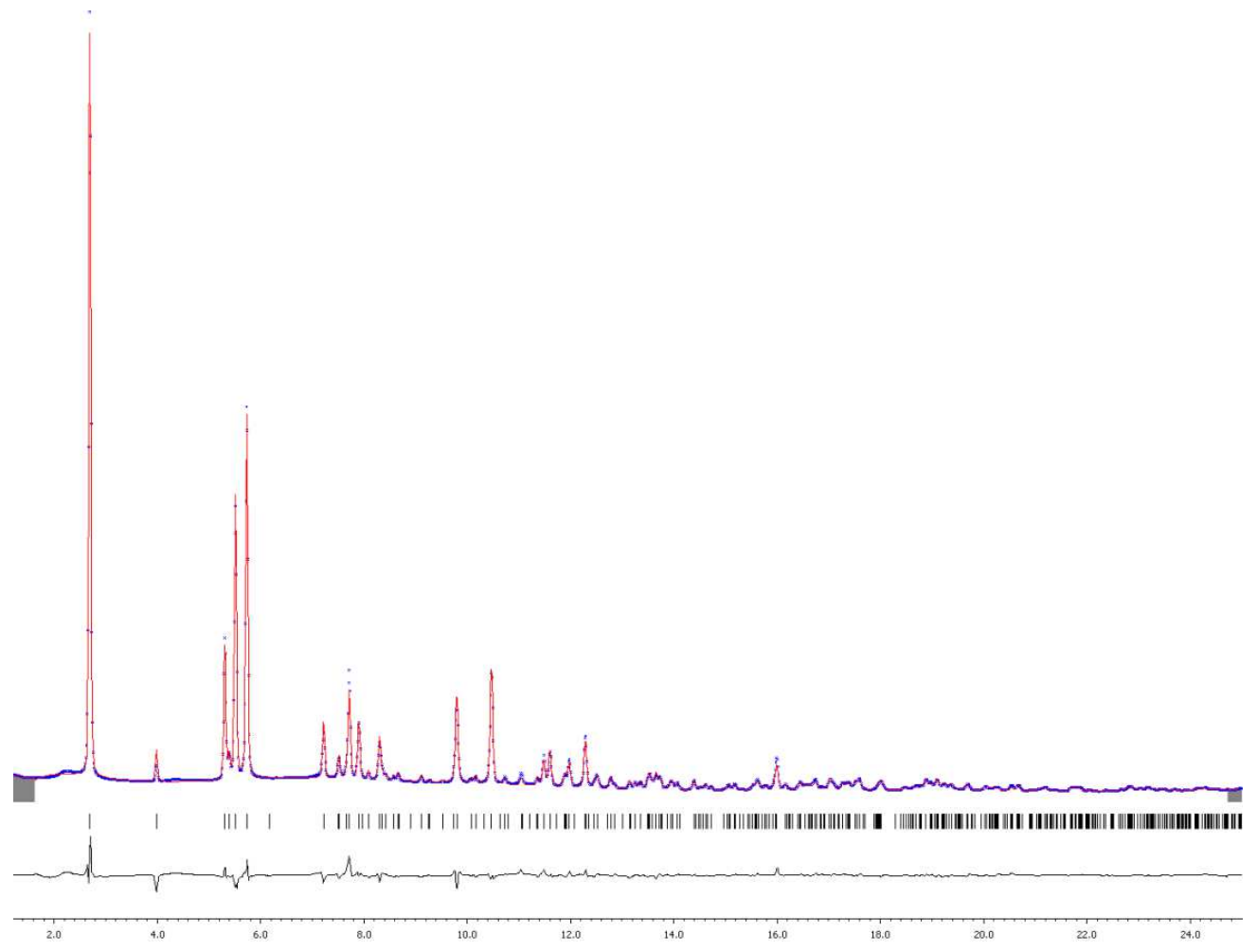

Figure 7S. Final Rietveld refinement plot for the PCN-200a sample: $\mathrm{R}_{\mathrm{p}}=3.92 \%, \mathrm{R}_{\mathrm{wp}}=5.51 \% \mathrm{R}_{\mathrm{exp}}=1.35 \%$ and $\mathrm{R}_{\text {bragg }}=4.72 \%$

\section{Refernce}

Petricek, V., Dusek, M., Palatinus L. (2006). Jana2006. Structure Determination Software Programs. Praha, Czech Republic.: Institute of Physics. 